\title{
Novel Deperming Protocols to Reduce Demagnetizing Time and Improve the Performance for the Magnetic Silence of Warships
}

\author{
Sang-Hyeon $\operatorname{Im}^{1}\left(\mathbb{D}\right.$, Ho-Yeong Lee ${ }^{2} \mathbb{D}$ and Gwan-Soo Park ${ }^{2, *}$ \\ 1 Department of Electrical Engineering, Dong Eui University, Busan 47340, Korea; ish@deu.ac.kr \\ 2 Department of Electrical Engineering, Pusan National University, Busan 47340, Korea; hyl@pusan.ac.kr \\ * Correspondence: gspark@pusan.ac.kr; Tel.: +82-51-2788
}

check for updates

Citation: Im, S.-H.; Lee, H.-Y.; Park, G.-S. Novel Deperming Protocols to Reduce Demagnetizing Time and Improve the Performance for the Magnetic Silence of Warships. Energies 2021, 14, 6295. https:// doi.org/10.3390/en14196295

Academic Editor: Andrea Mariscotti

Received: 19 August 2021

Accepted: 29 September 2021

Published: 2 October 2021

Publisher's Note: MDPI stays neutral with regard to jurisdictional claims in published maps and institutional affiliations.

Copyright: (c) 2021 by the authors. Licensee MDPI, Basel, Switzerland. This article is an open access article distributed under the terms and conditions of the Creative Commons Attribution (CC BY) license (https:// creativecommons.org/licenses/by/ $4.0 /)$.

\begin{abstract}
Magnetic silence of warships is necessary to prevent damage caused by the magnetic mines detecting the magnetic field of the warship. Anhysteretic and Deperm-ME protocols are used to reduce permanent magnetization among magnetic signals. However, they have some disadvantages. Therefore, this paper proposes an effective deperming protocol that is easily controlled and reduces the demagnetization time. A protocol composed of two Anhysteretic protocols is presented using the Preisach model to easily manage and ensure excellent performance. Each stage has its own advantages by considering the Preisach density distribution. In Stage 1, the existing magnetic history is erased, and the demagnetization time is reduced. In Stage 2, the demagnetization performance is improved. The effectiveness of the protocol was verified via simulations using the Preisach model and experiments using a specimen. When the proposed protocol was applied, the results were excellent when applying Anhysteretic and Deperm-ME. In addition, even if the number of magnetic fields was reduced by 4 and 8 in the proposed protocol, the demagnetization result was maintained. Therefore, if the proposed protocol is applied, excellent demagnetization results can be obtained and the time required to perform demagnetization can be reduced, thereby improving the operational capability of the warship.
\end{abstract}

Keywords: Anhysteretic deperm; Deperm-ME; demagnetization; Preisach model

\section{Introduction}

The magnetic signals of the warship consist of the induced magnetic field caused by the earth's magnetic field during operation [1-3], eddy currents by rolling [4], and the permanent magnetization generated during the manufacturing process. Most of the signals are the induced and the permanent magnetic fields. The induced magnetization changes according to the position, course, and azimuth of the ship under the earth's magnetic fields. The permanent magnetization was generated inside the warship, as shown in Figure 1a, owing to the external environment, such as the change of local magnetic properties by welding and cutting the hull of the ship during manufacturing. Since the magnetic mines installed in the sea detect these signals and attack the warship, the ship's magnetization has to be reduced to a certain magnitude. In particular, the permanent magnetization is less than $50 \%$ of the induced magnetization.

In general, demagnetization is performed for magnetic treatment of the warship, and then the induced magnetization is removed through the degaussing. A coil was wound around the warship to reduce permanent magnetization, as shown in Figure 1b, and the demagnetization was performed by applying continuously alternating and decreasing magnetic fields. These magnetic fields are called the deperming protocol. As the magnetic sensor installed outside the ship can measure the induced magnetic field, it can be eliminated by applying a canceling current to the coil installed inside the ship [2,3]. After the 
permanent magnetization is sufficiently reduced by demagnetization, the induced magnetization is more easily compensated by the degaussing coils with less power required.
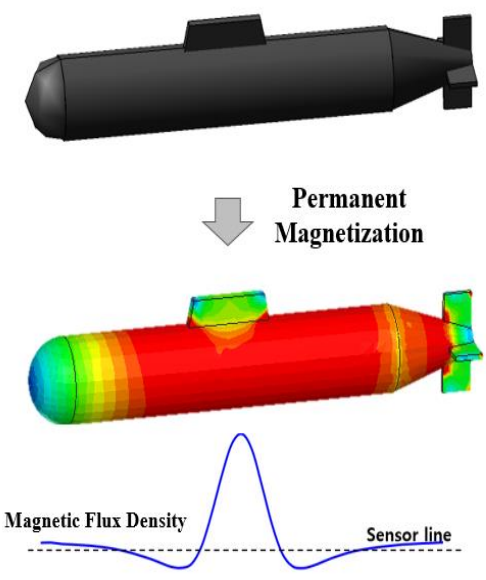

(a)
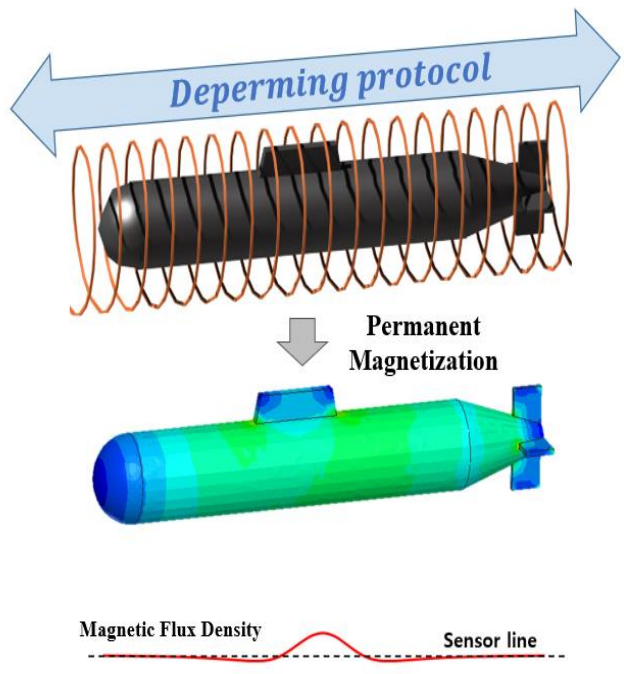

(b)

Figure 1. Schematic view of the demagnetization effects: (a) effect of the permanent magnetization after manufacturing the warship; (b) effect of the demagnetization.

The ship's magnetization consists of a Cartesian component, with mostly longitudinal and vertical components. In the case of demagnetization, it is generally used to reduce the longitudinal component. From the practical point of view, the result of demagnetization should reach less than half of the induced magnetic field component. The vertical component is usually compensated by degaussing coils but since a submarine does not have degaussing coils, it is magnetized in the opposite direction of the earth's magnetic field to eliminate the effect.

The most commonly used protocols for demagnetization are Anhysteretic Deperm, Deperm-ME, and Flash-D [5-7]. Flash-D is mainly used in submarines without degaussing coils, while others are used in warships equipped with degaussing coils. Several recent studies have shown that Deperm-ME performs better than Anhysteretic Deperm [8]. However, since complex control is required by the exponential function, it is not easy to use in the Navy. Therefore, a deperming protocol with better performance and easy control is needed. In addition, if the demagnetization time can be reduced, the operational capabilities of the warships can be improved.

This paper proposes an effective deperming protocol that is easy to control and can reduce the demagnetization time by using the Preisach model. The Preisach model can represent the hysteresis characteristics by considering the interactions between the magnetic particles inside the material. Therefore, it is suitable for demagnetization research. Moreover, in this paper, the influence of the earth's magnetic field is removed. There are two reasons. The first is that the effect is compensated by degaussing systems after demagnetization. Second, the magnitude of the earth's magnetic fields varies depending on the operation regions. Therefore, only an effective deperming protocol was studied in this paper.

The proposed protocol consists of two Anhysteretic protocols. The first protocol starts with a high magnetic field level to erase the previous history. Additionally, it operates in areas of low Preisach density, reducing the demagnetization time by decreasing unnecessary magnetic fields. The second protocol improves the demagnetization performance by drawing small traces in an area with high Preisach density distributions.

Simulations were performed using a program that combines the Preisach model and the Finite Element Method (FEM) to compare the proposed protocol with Anhysteretic and Deperm-ME. After applying each protocol, the distributions of the permanent mag- 
netization were analyzed. In addition, experiments were performed in a scaled-down Magnetic Treatment Facility (MTF) test room in which the earth's magnetic field and external influences were removed to verify the simulation results.

\section{Proposed Deperming Protocol Using the Preisach Model}

\subsection{Preisach Model}

The Preisach model was adopted in this research because it considers the coercive force and interactions between magnetic domains. The demagnetization process is also analyzed through the Jiles-Atherton model, which is one of the hysteresis modeling methods [9]. However, since it is difficult to consider the density distribution of magnetic materials, the Jiles-Atherton model is intuitively used for simulation rather than development of deperming protocols. On the other hand, the Preisach model calculates the amount of magnetization by integrating the density of hysterons corresponding to magnetic particles. As shown in Figure 2, the magnetic properties were represented by the density distribution of hysterons considering coercive force and interaction forces. Each hysteron is an element that can take only two values $((-1,1),(0,1)$-depending on the model design decision) [10].
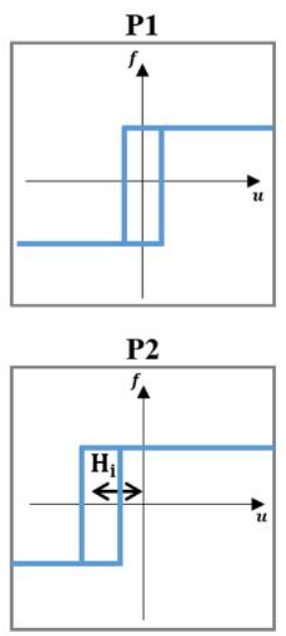
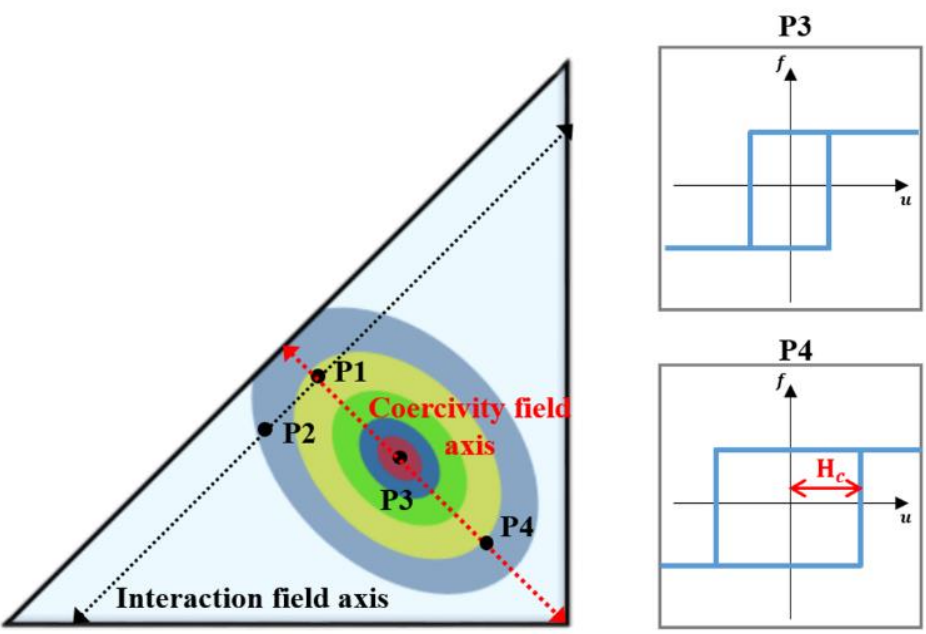

Figure 2. Schematic of Preisach density distribution according to coercivity field and interaction fields on the Preisach plane.

For example, there were hysterons with small coercive force and no interaction at P1. However, if there are interactions between hysterons, some hysterons will be shifted, such as those at P2. That is, along an interaction field axis, hysterons having the same coercive force had different interaction forces. Similarly, hysterons located on the same coercivity field axis had the same interaction force. Hysterons with larger coercive force were located at positions P3 and P4.

\subsection{Conventional Deperming Protocols}

Figure 3 shows the conventional deperming protocols: Anhysteretic Deperm and Deperm-ME. Anhysteretic Deperm is easy to control because it has a constant decrease as shown in Figure 3a. Thus, it is a demagnetization technique mainly used in the Navy. On the other hand, there are also disadvantages. The demagnetization performance is not as effective as that of the Deperm-ME protocol. Deperm-ME shows excellent performance according to exponential variation, as shown in Figure 3b [8]. However, there is reluctance in using it due to difficulties in control and variable determination and problems such as reliability verification. Therefore, it is necessary to study the demagnetization protocol that has the advantages of both protocols. 


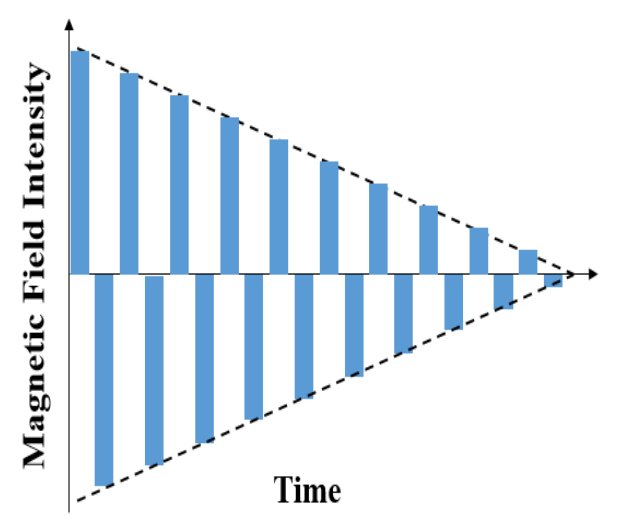

(a)

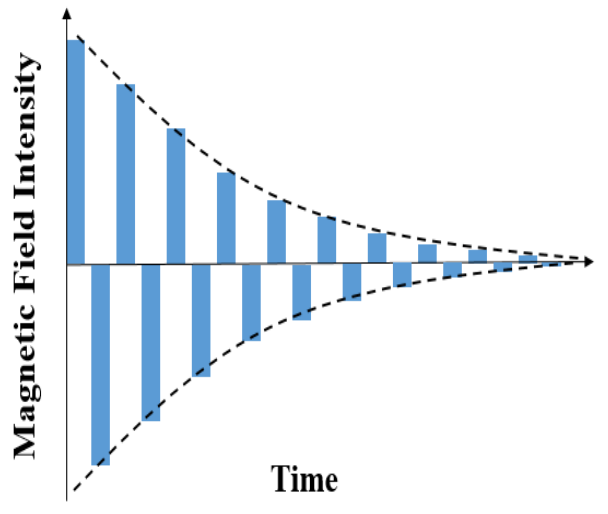

(b)

Figure 3. Schematic view of the conventional protocols: (a) Anhysteretic Deperm; (b) Deperm-ME.

\subsection{The Proposed Deperming Protocol Using the Preisach Model}

This paper proposes a new deperming protocol using Anhysteretic Deperm with high reliability and analyzes it using the Preisach model. It consists of two Anhysteretic Deperm protocols and has better performance than Anhysteretic Deperm and is easier to control than Deperm-ME. Figure 4 shows the process of determining the protocol.

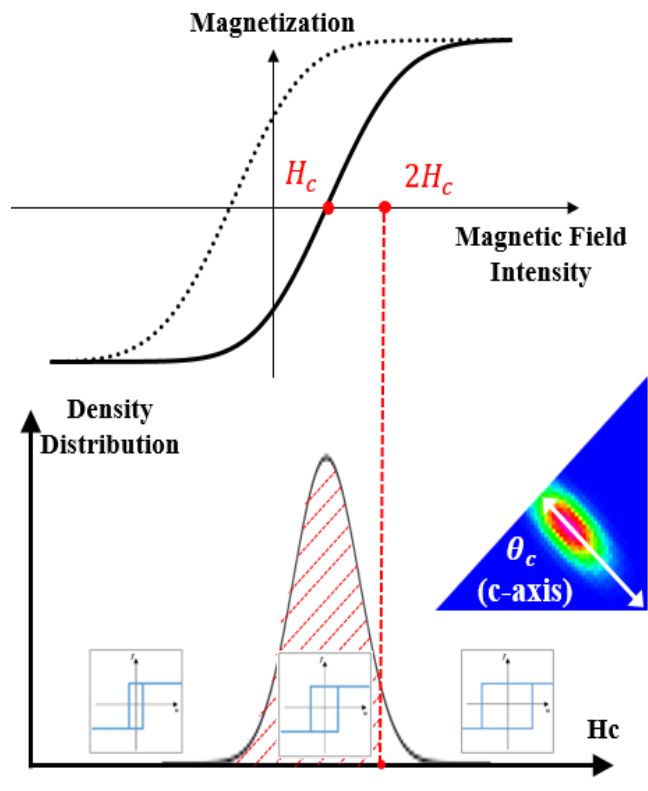

(a)

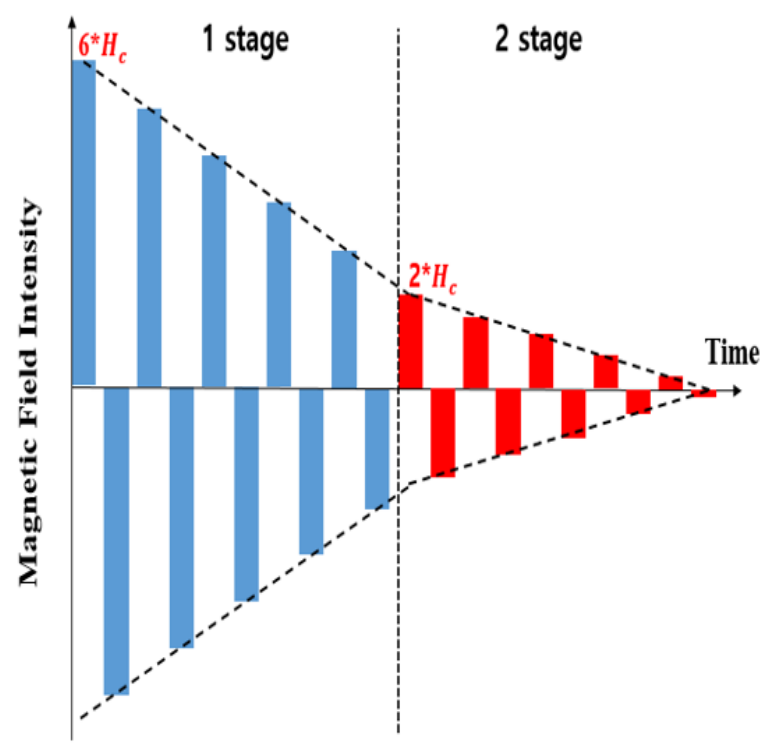

(b)

Figure 4. Process used to determine the proposed protocol: (a) the process; (b) schematic view of the proposed protocol.

In Stage 1, the initial magnetic field is approximately 6 times higher than the coercive force because the magnetic history of the warship should be erased even if it is largely magnetized by the external environment.

The second protocol starts with a magnitude that is twice the coercive force. When the magnetic flux density (B)-magnetic field strength $(\mathrm{H})$ curve is differentiated, it represents the density distribution of the Hc axis on the Preisach plane. Most of the particles are distributed in the region where the magnitude is less than twice the coercive force, as shown in Figure 4a. Therefore, the particles would mainly be demagnetized in Stage 2. Finally, the protocol is determined using the number of the applied magnetic fields and the magnitude of the initial and final magnetic fields as shown in Figure $4 \mathrm{~b}$.

The traces are drawn on the Preisach plane when the proposed protocol is applied, as shown in Figure 5a. As the variations are different in the two regions, the traces were large 
in Stage 1, and dense in Stage 2. The total magnetization remaining inside the warship is obtained using Equation (1), representing the sum of the densities in the area along the traces. If the demagnetization is well performed, the total magnetization would be close to zero. The hysteresis curve calculated according to the applied field is shown in Figure 5b.

$$
\text { Magnetization }=\iint_{S_{+}} p(a, b) d a d b+\iint_{S_{-}} p(a, b) d a d b
$$

where $S+S-$ are the Preisach planes divided by the traces, respectively, $a$ and $b$ are the coordinates, and $p(a, b)$ is the Preisach density.

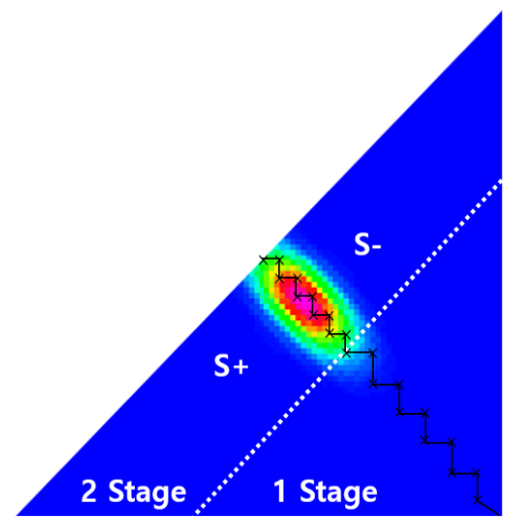

(a)

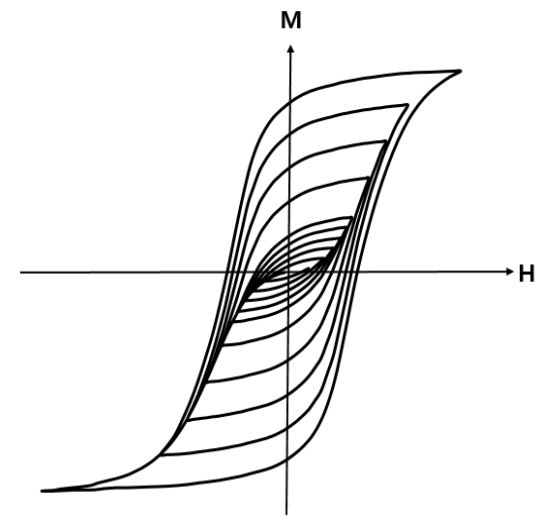

(b)

Figure 5. The traces on the Preisach plane following the proposed protocol: (a) the traces on the Preisach plane; (b) magnetization (M)-magnetic field strength $(\mathrm{H})$ curve.

Each stage has its advantages. In Stage 1, large magnetic fields are applied to erase the magnetic history. As a result, several traces are unnecessarily drawn in an area with low Preisach density distributions, as shown in Figure 6a. Since these traces do not affect the result of demagnetization, the number of magnetic fields can be reduced. This has the advantage of reducing the time for demagnetization. Figure $6 \mathrm{~b}$ shows the traces on the Preisach plane after removing two magnetic fields. Since this area has low density, the two regions divided by the traces on the plane and the corresponding total magnetization are similar. Therefore, in Stage 1, the demagnetization time can be reduced.

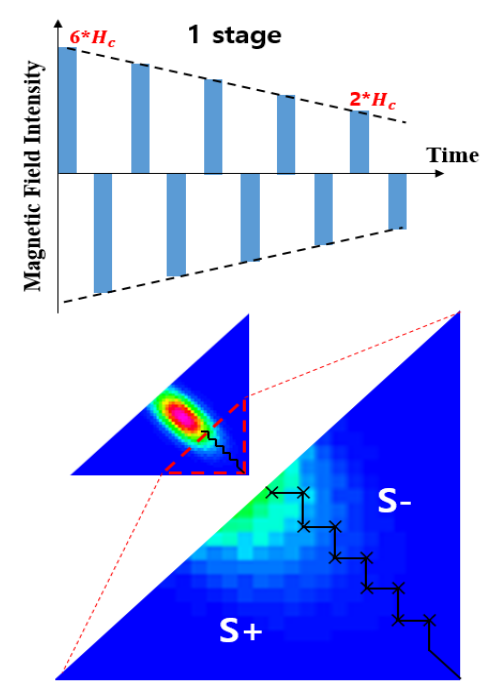

(a)

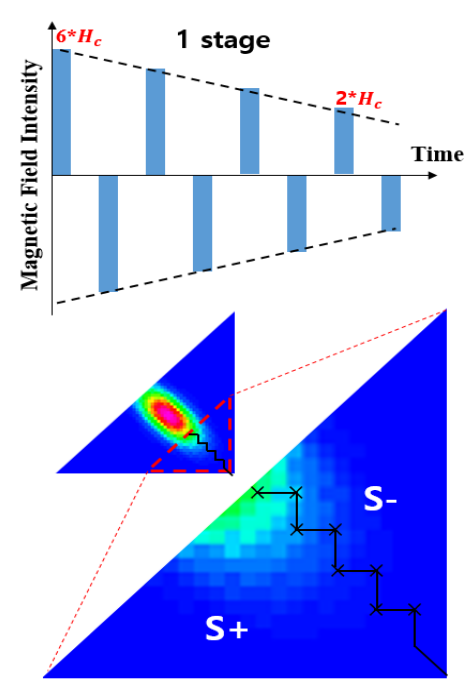

(b)

Figure 6. The traces of the Preisach plane in Stage 1 of the proposed protocols: (a) 10 magnetic fields; (b) 8 magnetic fields. 
Stage 2 plays an essential role in the demagnetization performance. Figure 7 shows the second protocol and the traces on the Preisach plane. Since it has minor variations and operates in an area with high Preisach density distribution, the Preisach plane is divided uniformly, allowing an effective demagnetization.

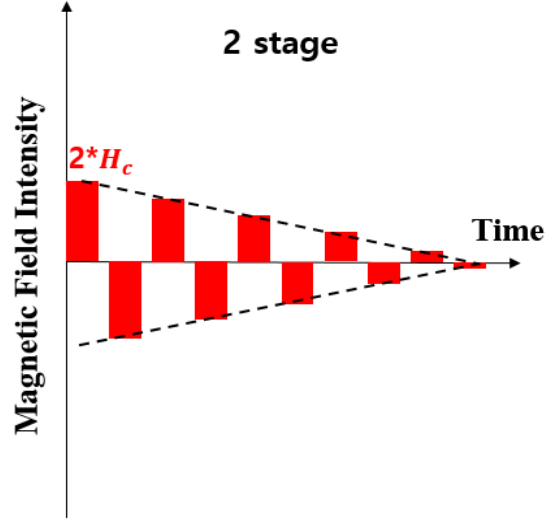

(a)

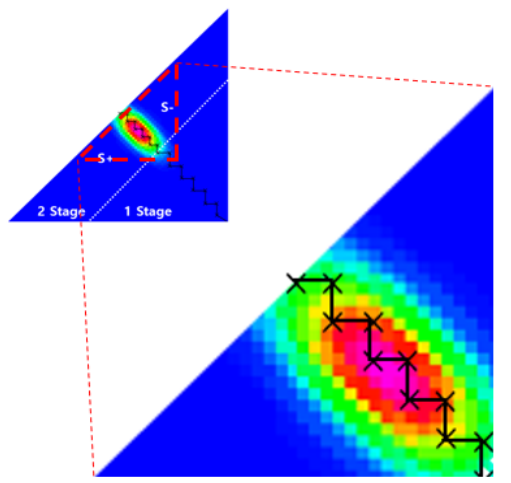

(b)

Figure 7. Schematic view of Stage 2 of the proposed protocols: (a) Stage 2 of the proposed protocol; (b) the traces on the Preisach plane.

Since the proposed protocol has both excellent performances of Deperm-ME and convenient control of Anhysteretic Deperm, it can be used in the real environment. In addition, it can reduce the demagnetization time.

\section{Simulation and Experiment Setup}

Simulations were performed through a program that combines the Preisach model and Finite Element Method (FEM) to verify the proposed protocol. In addition, experiments were conducted to verify the simulation results. Since the magnitude of the earth's magnetic field is dependent on the operation areas and the effect of the earth's magnetic field is compensated by degaussing after demagnetization, for the basic research of the deperming protocol, the effect of the earth's magnetic field was removed and the experiment was carried out in a Magnetic Treatment Facility.

Figure 8 shows a specimen and the deperming coil used in the simulations and experiments. The length, outer, and inner diameter of the specimen made of the Steel Plate Cold Commercial (SPCC) magnetic material were 298, 44, and $42 \mathrm{~mm}$, respectively. The demagnetizing coil was $557 \mathrm{~mm}$ long, $60.4 \mathrm{~mm}$ in diameter, and folded in 606 turns. The B-H curve of the SPCC and the Preisach density distribution are shown in Figure 9.

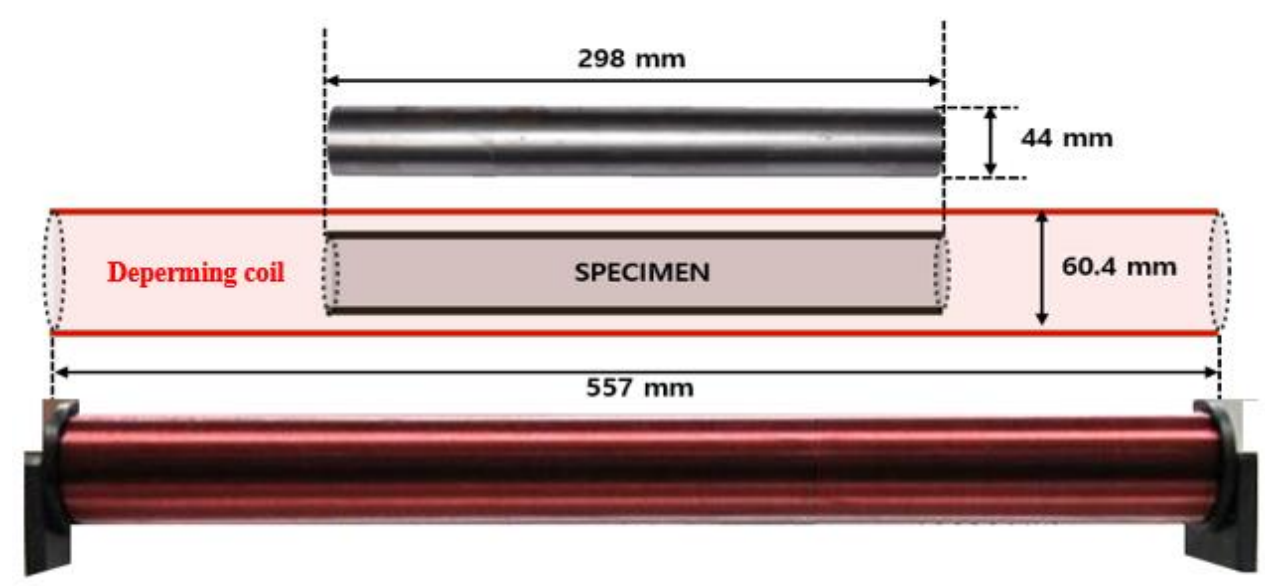

Figure 8. Model of the demagnetization experiment and simulation. 


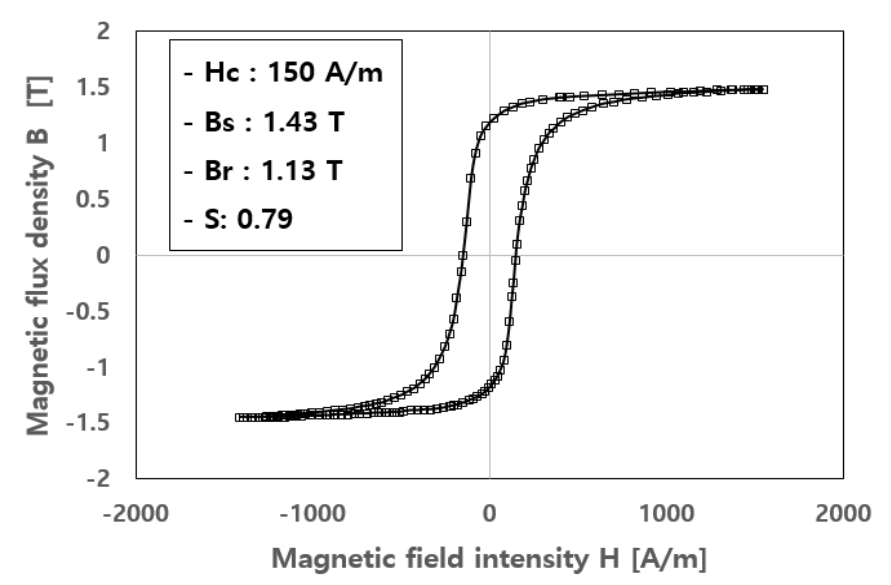

(a)

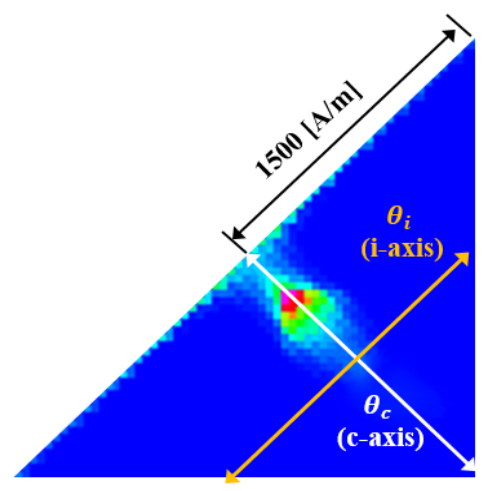

(b)

Figure 9. Magnetic properties of the SPCC: (a) B-H curve; (b) Preisach density distribution on the Preisach plane.

\section{Results and Discussion}

\subsection{Simulation Results}

Three protocols were applied to the coil for comparison (see Figure 10). The initial and final magnetic fields and the number of the applied magnetic fields were the same. Each magnetic field lasts for $5 \mathrm{~s}$, with a rest period of $3 \mathrm{~s}$. Anhysteretic has a constant decrease of $111.1 \mathrm{~A} / \mathrm{m}$. On the other hand, in the proposed protocol, the reduction is 191.2 in Stage 1, and is constantly decreased by $39.5 \mathrm{~A} / \mathrm{m}$ in Stage 2 . The proposed protocol was determined through the process shown in Figure 4.

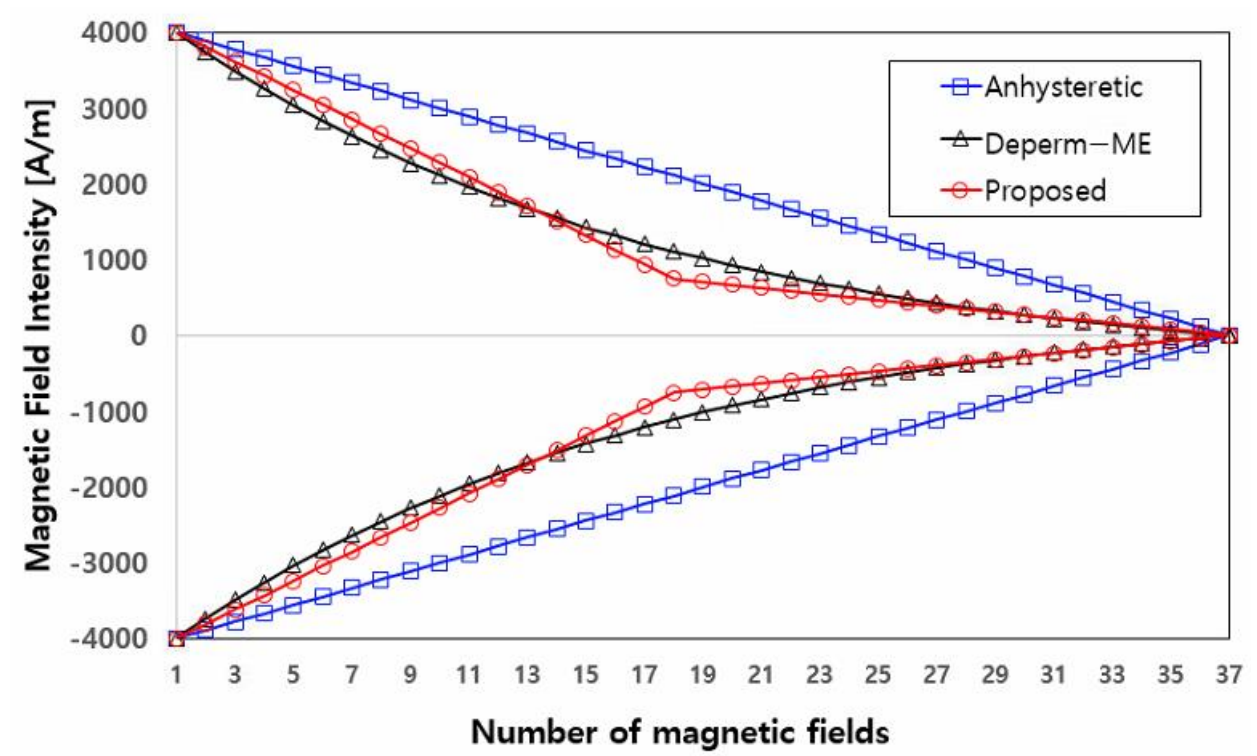

Figure 10. Three types of deperming protocols.

Figure 11 shows the distribution of the internal magnetization after the demagnetization process performed according to the protocols. After applying the Anhysteretic protocol, a large amount of permanent magnetization remains inside, as shown in Figure 11a. The results obtained through the Deperm-ME protocol were reduced compared to those obtained with the Anhysteretic Deperm protocol. However, as shown in Figure 11c, the internal magnetization after using the proposed protocol was lower than that obtained with the other two protocols. Therefore, the demagnetization performance of the proposed protocol is the best among the protocols. 


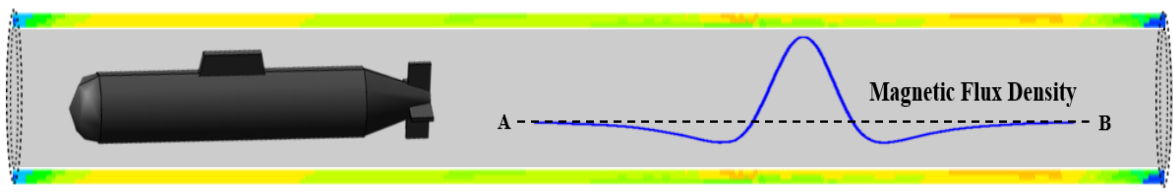

(a)

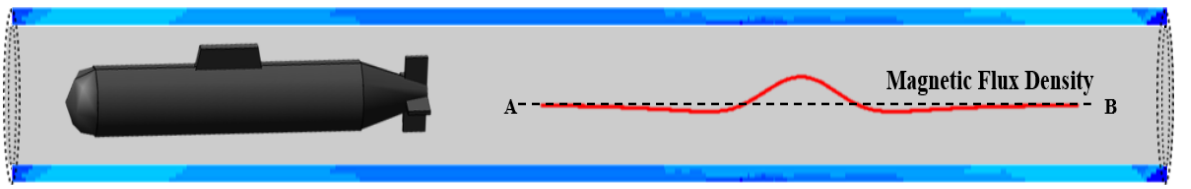

(b)

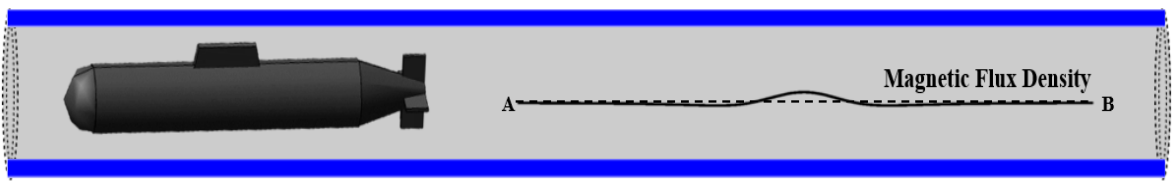

(c)

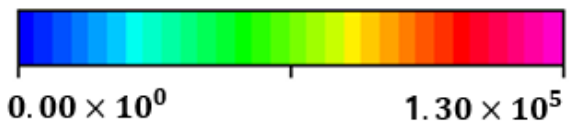

(d)

Figure 11. Simulated distribution of the residual magnetization according to the protocols: (a) Anhysteretic Deperm; (b) Deperm-ME; (c) proposed protocol; (d) contour bar.

\subsection{Experiment Results}

Figure 12 shows the experimental setup. A SPCC specimen was placed inside the $X$ coil and three protocols were applied to the $X$ coil. The magnetic flux density was measured with a magnetic sensor (Mag690, Bartington Instruments, Witney, England) to confirm the demagnetization results. The sensor that was positioned $18 \mathrm{~cm}$ below it in the vertical direction measured the magnetic flux density while moving the specimen on the aluminum rail. The $Z$ coil was not used in this study since it is used in the Flash-D protocol for submarines. All the devices around the flux-gate magnetic sensor were made of non-magnetic aluminum to minimize the effects of the external magnetic fields.

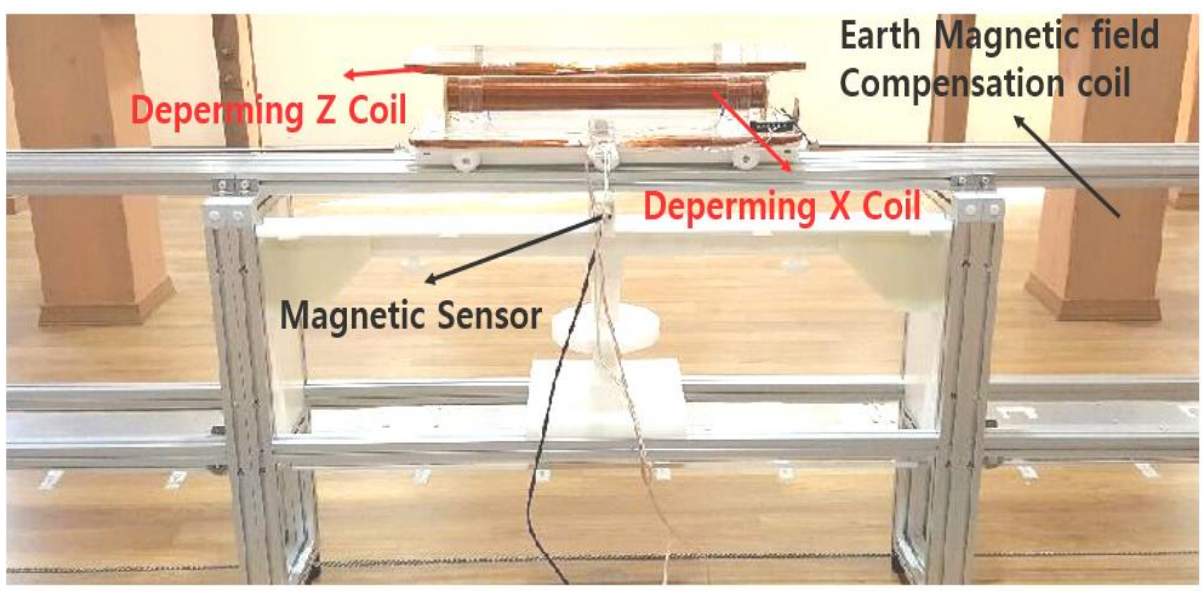

Figure 12. Sensing system for deperming.

Figure 13 and Table 1 show the results of the experiment. Since all the protocols used the same number of applied fields, the operating time was the same. When the Deperm-ME protocol was applied, the magnetic flux density was $66.5 \%$ lower than that obtained with the Anhysteretic Deperm protocol. The proposed protocol reduced the signal by $95.5 \%$. 


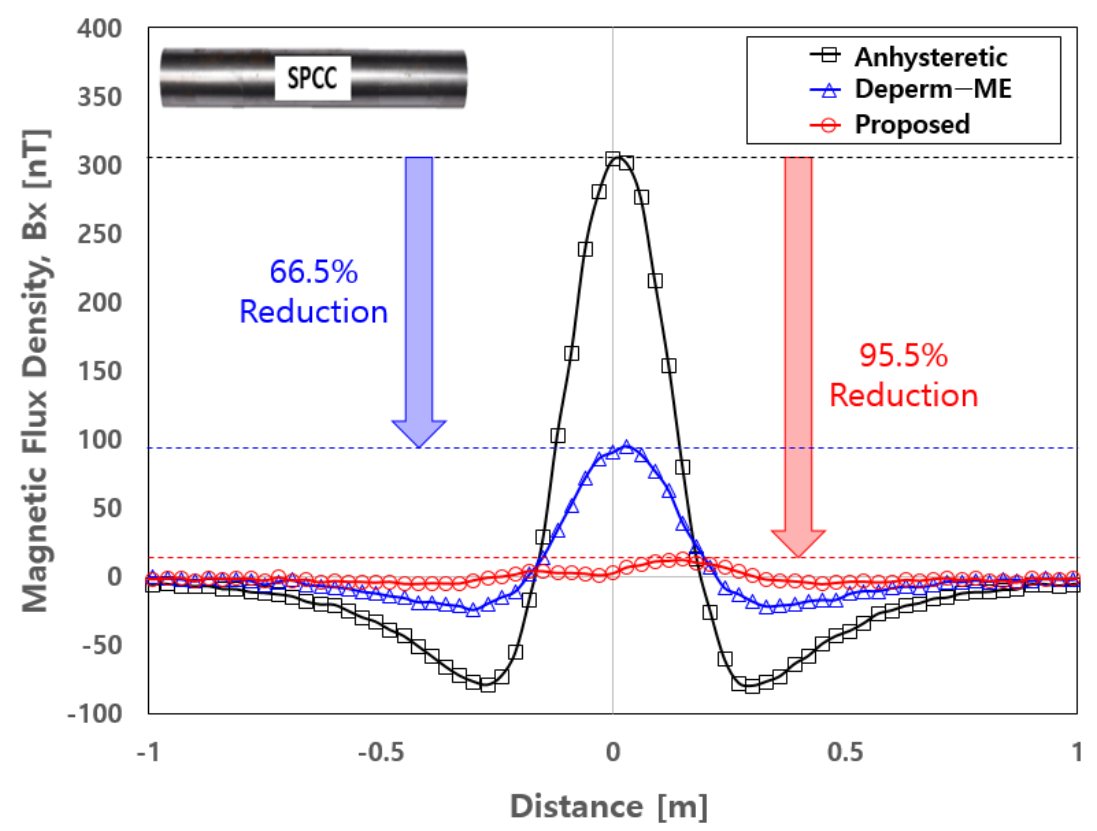

Figure 13. Experimental results of the SPCC following the deperming protocols.

Table 1. Measured magnetic flux density of the SPCC following the protocols.

\begin{tabular}{cccc}
\hline Protocols & Shot Numbers & Time & $\begin{array}{c}\text { Magnetic Flux Density } \\
\text { (Reduction Rate) }\end{array}$ \\
\hline Anhysteretic & 36 & $288 \mathrm{~s}$ & $304.0 \mathrm{nT}$ \\
Deperm-ME & 36 & $288 \mathrm{~s}$ & $94.9 \mathrm{nT}(33.5 \%)$ \\
Proposed & 36 & $288 \mathrm{~s}$ & $12.2 \mathrm{nT}(4.5 \%)$ \\
\hline
\end{tabular}

Additional experiments were performed to verify whether the proposed protocol could reduce the demagnetization time. Stage 2 is fixed in the proposed protocol, and the number of applied magnetic fields in Stage 1 is reduced by 4 and 8 magnetic fields. Figure 14 and Table 2 show the results of the experiments.

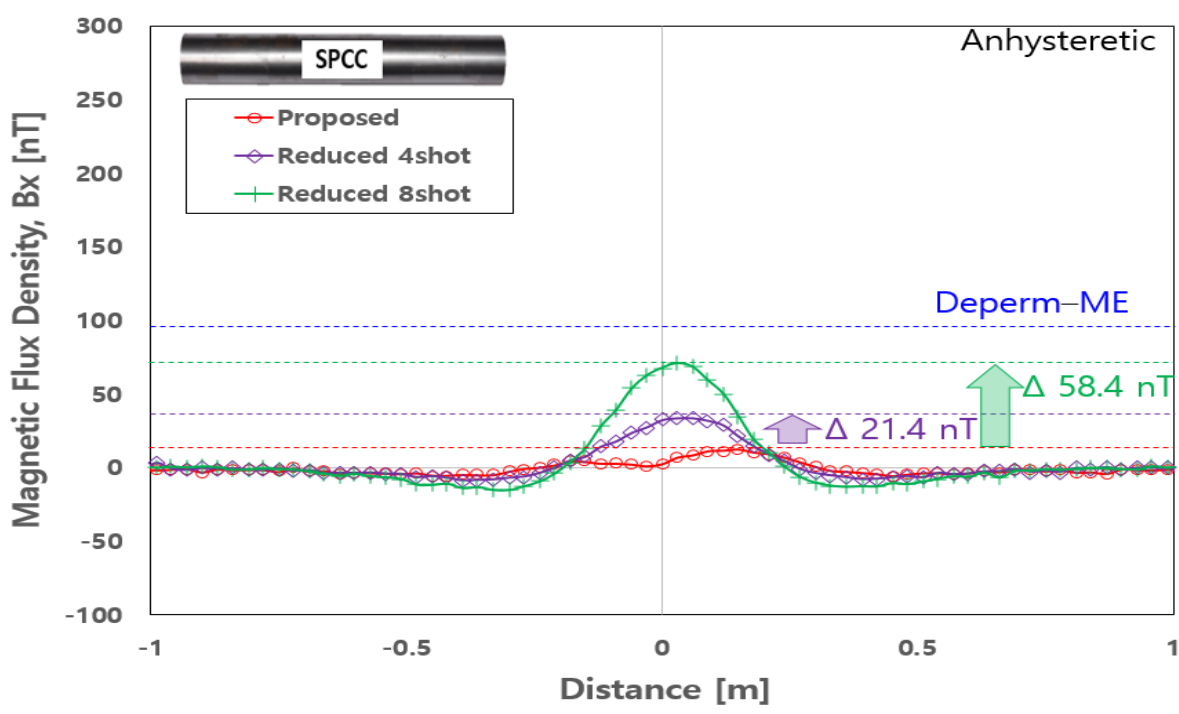

Figure 14. Experimental results obtained with a reduced number of magnetic fields. 
Table 2. Measured results of the SPCC by reducing the demagnetization time.

\begin{tabular}{cccc}
\hline Protocols & Shot Numbers & $\begin{array}{c}\text { Time } \\
\text { (Reduction Rate) }\end{array}$ & $\begin{array}{c}\text { Magnetic Flux } \\
\text { Density }\end{array}$ \\
\hline Deperm-ME & 36 & $288 \mathrm{~s}$ & $94.9 \mathrm{nT}$ \\
Proposed & 36 & $288 \mathrm{~s}$ & $12.2 \mathrm{nT}$ \\
Reduced 4 shot & 32 & $256 \mathrm{~s}$ & $33.6 \mathrm{nT}$ \\
Reduced 8 shot & 28 & $(88.8 \%)$ & $70.6 \mathrm{nT}$ \\
\hline
\end{tabular}

When 4 and 8 magnetic fields were reduced, the flux density increased by 21.4 and $58.4 \mathrm{nT}$, respectively. As the number of magnetic fields decreased, the signal increased. However, the performance was better than that of Deperm-ME. Furthermore, the demagnetization times were reduced by $11.1 \%$ and $22.2 \%$, respectively. Therefore, the proposed protocol has effective performance and reduces the demagnetization time and is easy to control.

\section{Conclusions}

This paper proposed a novel deperming protocol consisting of two Anhysteretic protocols using the Preisach model. In Stage 1, the previous history was erased through a large initial magnetic field. Due to the low density of the region, unnecessary magnetic fields can be removed. In Stage 2, due to the high density distribution, the performance was improved by drawing the traces uniformly with a slight variation.

The simulations and experiments show that the proposed protocol has better performance than the Anhysteretic and Deperm-ME protocols. In addition, the demagnetization time can be reduced by up to $22 \%$ while maintaining excellent performance.

Author Contributions: Conceptualization, S.-H.I. and G.-S.P.; methodology, S.-H.I. and G.-S.P.; data curation, S.-H.I. and H.-Y.L.; writing—original draft preparation, S.-H.I.; writing—review and editing, S.-H.I.; visualization, S.-H.I.; supervision, G.-S.P. All authors have read and agreed to the published version of the manuscript.

Funding: This research was supported by Basic Science Research Program through the National Research Foundation of Korea (NRF) funded by the Ministry of Education (2020R1I1A1A01072534).

Conflicts of Interest: The authors declare no conflict of interest.

\section{References}

1. Modi, A.; Kazi, F. Electromagnetic Signature Reduction of Ferromagnetic Vessels Using Machine Learning Approach. IEEE Trans. Magn. 2019, 55, 6000606. [CrossRef]

2. Norgen, M.; He, S. Exact and explicit solution to a class of degaussing problems. IEEE Trans. Magn. 2000, 36, 308-312. [CrossRef]

3. Raveendra, R.; Varm, A. Design of Degaussing System and Demonstration of Signature Reduction on Ship Model through Laboratory Experiments. Phys. Procedia 2014, 54, 174-179.

4. Tarr, P.B. Method of Measuring Magnetic Effects Due to Eddy Currents. U.S. Patent 4648041A, 3 March 1987.

5. TBynes, M.; Russel, G.J.; Biley, A. Comparison of stepwise demagnetization techniques. IEEE Trans. Magn. 2002, 38, $1753-1758$.

6. Wang, R.F.; Li, J.; McConville, W.; Nisoli, C.; Ke, X. Demagnetization protocols for frustrated interacting nanomagnet arrays. J.Appl. Phys. 2007, 101, 9J104. [CrossRef]

7. Ju, H.S.; Chung, H.J.; Im, S.H.; Jeong, D.W.; Kim, J.W.; Lee, H.B.; Park, G.S. Efficient Deperming Protocols Based on the Magnetic Properties in Demagnetization Process. IEEE Trans. Magn. 2015, 51, 7301704. [CrossRef]

8. Ju, H.S.H.W.; Chung, H.J.; Park, G.S. A Study on the Deperm Procols Considering Demagnetizing Field of a Ferromagnetic Material. J. Magn. 2014, 19, 43-48. [CrossRef]

9. Mayergoyz, I.D.; Friedman, G. Generalized Preisach model of hysteresis. IEEE Trans. Magn. 1988, 24, 212-217. [CrossRef]

10. Birsan, M. Simulation of a Ship's Deperming Process Using the Jiles-Atherton Model. IEEE Trans. Magn. 2021, 57, 7300407. [CrossRef] 